\title{
Exceso de mortalidad por COVID-19 en la provincia más poblada de Argentina durante 2020
}

\author{
Excess mortality from COVID-19 in the most populated province of Argentina during 2020
}

\section{Comentado de:}

Pesci S, et al. Rev Argent Salud Pública. 2021;13:1-91

\section{Objetivos}

Determinar el impacto de la pandemia por COVID-19 sobre el exceso de mortalidad (EM) ocurrido en la provincia de Buenos Aires, Argentina.

\section{Diseño y lugar}

Estudio descriptivo, retrospectivo, de base poblacional, realizado en la provincia de Buenos Aires, Argentina.

\section{Fuentes de información}

Los autores de esta investigación realizaron una comparación entre la distribución de las muertes ocurridas durante las semanas epidemiológicas (SE) 1 a 53 de 2020 y aquellas registradas en el mismo período del quinquenio anterior (2015 a 2019), con el fin de determinar la presencia de EM en la provincia de Buenos Aires. El estudio se nutrió de los registros de defunciones de esta jurisdicción y del Departamento de Estadísticas Vitales y Demográficas para el periodo 2015 a 2019, mientras que para el periodo 2020 utilizó datos del Registro Provincial de las Personas. Es decir, se combinaron datos cerrados y consolidados de mortalidad con aquellos que se iban recolectando en el año en curso, que se incorporarían a las estadísticas vitales al año siguiente.

\section{Medición de resultados principales y análisis}

EI EM comprendió las defunciones por todas las causas que ocurrieron en la provincia de Buenos Aires, sin discriminar la residencia de origen. El cálculo de EM fue realizado mediante las estimaciones de P-score (Fórmula 1) y el límite superior del intervalo de confianza (IC) del $95 \%$ de las defunciones durante 2015 a 2019 (Fórmula 2) (ver Tabla 1). Estos resultados expresan el
EM como la diferencia entre el número anual de muertes de 2020 y el promedio de muertes ocurridas para el mismo periodo en los cinco años previos.

Tabla 1. Fórmulas empleadas para el cálculo del exceso de mortalidad en el estudio. Nota: * utilizando el límite superior del intervalo de confianza (IC) $95 \%$ del promedio histórico de las defunciones sucedidas entre 2015 y 2019

\begin{tabular}{|l|l|}
\hline Fórmula 1 & $\begin{array}{l}\text { P-Score }=(\text { mortalidad observada en } 2020 \text { - morta- } \\
\text { lidad promedio 2019 a 2015) / mortalidad promedio } \\
2019 \text { a } 2015\end{array}$ \\
\hline Fórmula 2 & $\begin{array}{l}\text { EM (IC 95\%) }=(\text { mortalidad observada en } 2020 \text { - mor- } \\
\text { talidad 2019 a 2015*)/ mortalidad 2019 a 2015* }\end{array}$ \\
\hline
\end{tabular}

Los resultados de EM fueron discriminados por sexo y grupo etario. Además, los autores realizaron un análisis comparativo con los valores de EM registrados durante las semanas epidemiológicas 1 a 53 en otros países de América y Europa. Por último, los autores evaluaron la relación entre el EM y las muertes confirmadas por COVID-19, para estimar el porcentaje de reemplazo de causa de manera comparativa entre países y regiones.

\section{Resultados Principales}

La diferencia en el número de muertes entre las medias de la serie histórica 2015 a 2019 y 2020 en la provincia de Buenos Aires fue de 15.792 defunciones. Esto equivale a un EM por P-score de $12,15 \%$ sobre el promedio basal $(90,02$ defunciones extra por cada 100.000 habitantes), y un EM de 7,59\% si se considera como línea de base el límite superior del IC $95 \%$ para la serie histórica (ver Tabla 2).

Tabla 2. Serie histórica 2015 a 2019 y defunciones en 2020 con ocurrencia en la provincia de Buenos Aires, Argentina. Nota: IC: intervalo de confianza

\begin{tabular}{|l|c|c|c|c|c|c|}
\hline Meses & $\begin{array}{l}\text { Número de } \\
\text { defunciones } \\
\text { (2015 a 2019) }\end{array}$ & $\begin{array}{l}\text { Límite supe- } \\
\text { rior del IC } \\
95 \% \text { del } \\
\text { número de } \\
\text { defunciones } \\
(2015 \text { a 2019) }\end{array}$ & $\begin{array}{l}\text { Número de } \\
\text { defunciones } \\
(2020)\end{array}$ & $\begin{array}{l}\text { Muertes por } \\
\text { COVID-19 } \\
\text { (2020) }\end{array}$ & $\begin{array}{l}\text { Diferencia en } \\
\text { el número de } \\
\text { defunciones }\end{array}$ & $\begin{array}{l}\text { Exceso de } \\
\text { mortalidad, } \\
\text { P-score }\end{array}$ \\
\hline $\begin{array}{l}\text { Enero a } \\
\text { Diciembre }\end{array}$ & 129.982 & 135.495 & 145.774 & 23.405 & 15.792 & $12,15 \%$ \\
\hline
\end{tabular}

Al estratificar por grupo de edad y sexo, el estudio reportó que el EM asumió valores negativos entre los 0 y 29 años en el sexo femenino, y entre los 0 y 39 años en el masculino. Asimismo, hubo una diferencia promedio de EM en el sexo masculino de 0,38 puntos sobre el femenino; y entre los 40 y los 90 años, el EM entre varones fue superior al de las mujeres. Esto explica que, si bien el mayor porcentaje de EM en el sexo femenino se observó en el grupo de 40 a 49 años de edad (35,59\%), el mayor porcentaje global de EM (sin distinción por sexo) se observó en el grupo de 70 a 79 años (38,63\%), el mismo grupo etario en el que se observó el mayor porcentaje de EM entre hombres (41,01\%).

EI EM en la provincia de Buenos Aires presentó valores menores a los de España, Estados Unidos, Inglaterra y el resto de los países latinoamericanos analizados, aunque por encima de lo reportado por Portugal, Países Bajos, Suiza, Escocia, Francia, Alemania, Dinamarca, Noruega y Nueva Zelanda, entre otros.

Las muertes confirmadas por COVID-19 en la provincia de Buenos Aires superaron el EM en una relación porcentual de $130,51 \%$, en contraste con países de la región como Ecuador, México y Perú, en los que el EM supera ampliamente a las muer- 
tes confirmadas por COVID-19.

\section{Conclusiones}

Los resultados de este estudio mostraron la presencia de un EM en la provincia estudiada durante el año 2020, que alcanza un $12,15 \%$ respecto al promedio del quinquenio previo y afecta en forma diferencial a la población según el sexo y la edad.
Las muertes por COVID-19 en la provincia de Buenos Aires superaron el EM, lo que podría indicar la existencia de un reemplazo de otras causas de defunción.

Conflicto de interés de los autores / Fuentes de financiamiento: No informados en el artículo original.

\section{Comentario}

EI EM hace referencia al número de muertes por todas las causas en exceso que suceden durante un periodo de crisis, en comparación con lo que hubiéramos esperado observar en condiciones consideradas normales. Este estudio evidencia la presencia de EM durante el año 2020 en la provincia de Buenos Aires, en consonancia con resultados obtenidos por estudios similares ${ }^{2}$.

Una de sus limitaciones incluye el haber empleado datos no consolidados por parte del Departamento de Estadísticas Vitales del Ministerio de Salud provincial para el periodo 2020, lo que impidió realizar análisis más exhaustivos sobre las causas específicas de defunción durante la pandemia.

Puede llamar la atención que algunos de los países con los que se comparó la provincia argentina analizada, las muertes confirmadas por COVID-19 representaron un número sustancialmente menor que las cifras de EM. Recordemos que el EM es un indicador que permite identificar no solo las muertes confirmadas por COVID-19, sino también aquellas muertes por COVID-19 que no fueron correctamente diagnosticadas o registradas, así como otras muertes que, si bien no se debieron de manera directa al virus SARS-CoV-2, estuvieron relacionadas con las condiciones que la pandemia generó en el sistema de salud (tales como la atención con retraso de otros problemas de salud, la saturación de los servicios de internación y guardia, o el propio aislamiento social, entre otras). La observación de que en la provincia de Buenos Aires las muertes confirmadas por COVID-19 superaron al EM permite inferir que no hubo un subregistro de las muertes confirmadas por esta causa en esta jurisdicción.

Podemos asumir que el EM constituye un indicador más completo y sólido que el número de muertes por COVID-19 a la hora de medir el impacto real de la pandemia. Sin embargo, debemos tener en cuenta que el análisis de este indicador no permite inferir causalidad, atribuyendo el EM de manera directa al efecto de la propagación del virus SARS-CoV-2, dada la composición multifactorial de factores intervinientes y la complejidad de sus interacciones que determinan la mortalidad de la población, y que, en el mundo real, exceden el concepto epidemiológico clásico de pandemia.

\section{Conclusiones del comentador}

La estimación del EM se ha transformado en un indicador cada vez más utilizado para valorar el impacto de la pandemia de COVID-19, generando que los sistemas de vigilancia y seguimiento sean cada vez más importantes a la hora de tomar decisiones gubernamentales para afrontar esta crisis, aunque este tipo de estudios descriptivos no permite inferir causalidad.

Mauricio Pablo Kemnitz [ Hospital Italiano de Buenos Aires, Servicio de Medicina Familiar y Comunitaria. mauricio.kemnitz@hospitalitaliano.org.ar ]

Kemnitz M. Exceso de mortalidad por COVID-19 en la provincia más poblada de Argentina durante 2020. Evid Actual Pract Ambul. $2021 ; 24(4)$ :e002165. Available from: https://dx.doi.org/10.51987/EVIDENCIA.V25I1.6989. Comentado de: Pesci S, et al. Exceso de mortalidad por la pandemia del COVID-19 durante 2020 en la provincia de Buenos Aires, Argentina. Rev Argent Salud Pública. 2021;13:1-9.

\section{Referencias}

1. Pesci S, Marín L, Wright R, et al. Exceso de mortalidad por la pandemia del COVID-19 durante 2020 en la provincia de Buenos Aires. Argentina Rev Argent Salud Pública. 2021;13:1-9.

2. Sanmarchi F, Golinelli D, Lenzi J, et al. Exploring the Gap Between Excess Mortality and COVID-19 Deaths in 67 Countries. JAMANetwOpen. 20211;4(7):2117359-2117359. Available from: 10.1001/jamanetworkopen.2021.17359. 